\title{
Contraception for women with diabetes: challenges and solutions
}

\author{
This article was published in the following Dove Press journal: \\ Open Access Journal of Contraception \\ 3 March 2016 \\ Number of times this article has been viewed
}

\section{Ann Robinson \\ Chidiebere Nwolise \\ Jill Shawe}

School of Health Sciences, Faculty of Health and Medical Sciences, University of Surrey, Surrey, UK
Correspondence: Ann Robinson School of Health Sciences, Faculty of Health and Medical Sciences, University of Surrey, Guildford, Surrey GU2 7XH, UK

Tel +44 I4836867I3

Email a.robinson@surrey.ac.uk
Abstract: Diabetes mellitus (DM), the most common of metabolic disorders, is a global public health concern. Numbers are rising with 383 million adults currently diagnosed with DM and another 175 million as yet undiagnosed. The rise in cases includes increasing numbers of women of a reproductive age whose reproductive health and contraception need careful consideration. Unintended pregnancy with poor glycemic control at the time of conception increases the chance of adverse pregnancy outcomes including stillbirth, congenital abnormalities, and perinatal mortality. In order to minimize complications, safe and effective contraception is paramount for all women with DM. This is a challenge as women have been found to be reticent to ask for advice, appear to lack understanding of risks, and are less likely to be using contraception than women without DM. The World Health Organization has developed Medical Eligibility Criteria to guide contraceptive choice. Women with DM without complications can choose from the full range of contraceptive methods including hormonal contraception as the advantages of use outweigh any risk. Women with diabetic complications may need specialist advice to assess the risk-benefit equation, particularly in respect of hormonal contraception. Women should be aware that there is no restriction to the use of oral and copper intrauterine emergency contraception methods. There is a need for an integrated approach to diabetes and reproductive health with improved communication between women with DM and their health care providers. Women need to be aware of advice and services and should make their own choice of contraception based on their needs and associated risk factors. Practitioners can offer nonjudgmental guidance working in partnership with women. This will enable discussion of risks and benefits of contraceptive methods and provision of advice dedicated to improving overall health and well-being.

Keywords: diabetes mellitus, gestational diabetes, contraception, education, preconception counseling

\section{Introduction}

Diabetes mellitus (DM), the most common of metabolic disorders, ${ }^{1}$ is a global public health concern. Three hundred and eighty three million adults are currently diagnosed with DM and another 175 million are estimated to be living with the condition but are as yet undiagnosed. ${ }^{2}$ By 2035, numbers are set to increase by 55\% with 592 million people becoming affected worldwide. ${ }^{2}$ The rise in cases includes increasing numbers of women of reproductive age whose reproductive health and contraceptive needs must be carefully considered as DM has serious implications for pregnancy. It is the most common medical condition complicating pregnancy and affects up to $10 \%$ of women of a reproductive age in developed countries. ${ }^{3}$ 
There are three main types of DM (Table 1). Type 1 DM is an autoimmune, chronic condition occurring in both adults and children, often before 40 years of age, and accounting for $\sim 10 \%$ of cases in adults and $98 \%$ of cases in children. ${ }^{4}$ In type $1 \mathrm{DM}$, the primary beta cells of the pancreas do not produce insulin resulting in hyperglycemia. The propensity to develop autoimmune diseases, such as diabetes, may be familial. Treatment involves careful glucose monitoring and administration of insulin.

Type 2 DM, accounting for $\sim 90 \%$ of cases in adults and $2 \%$ of cases in children, ${ }^{4}$ is sometimes known as mature onset diabetes. This occurs when the amount of insulin produced by the pancreatic beta cells is insufficient or if the body develops a resistance to the insulin produced. As this condition is often associated with obesity, treatment involves diet and exercise, oral medication, and occasionally insulin. Recently, more cases of type 2 DM have been diagnosed in younger age groups due to increasing obesity. ${ }^{1}$

Gestational DM (GDM) develops during pregnancy in women without previously diagnosed DM. It is caused by increased blood glucose levels in pregnancy and is treated with diet or insulin. GDM usually disappears after pregnancy but women are at risk of developing type $2 \mathrm{DM}$ in the future.

This paper will review some of the challenges for women with DM in relation to contraception and will suggest solutions guided by current evidence.

\section{Methods}

To update previous searches for new primary research and reviews, we searched MEDLINE (754 articles), PsycINFO (95 articles), CAB Abstracts (451 articles), CINAHL (174 articles), Maternity and Infant Care (49 articles), Web of Science (53 articles), British Nursing Index (35 articles), Embase (150 articles), and ScienceDirect ( 76 articles) from 2008 to 2015. The following terms were used for MEDLINE

Table I Prevalence of diabetes

\begin{tabular}{ll}
\hline Prevalence of diabetes (20 I 4) $)^{2,4}$ \\
$\begin{array}{ll}\text { Adults aged 20-79 } & 387 \text { million people were affected in 20I4. This } \\
\text { years worldwide } & \text { figure was calculated to rise to } 592 \text { million by } 2035^{2} \\
\text { Undiagnosed } & \text { I79 million people were calculated to have } \\
\text { type 2 diabetes } & \text { undiagnosed type 2 Diabetes }\end{array}$ \\
$\begin{array}{ll}\text { Type I and type 2 } \\
\text { diabetes }\end{array}$ & $\begin{array}{l}\text { Type I diabetes - I0\% of those affected } \\
\text { Global estimations } 2 \text { diabetes - 90\% of those affected }\end{array}$ \\
& $\begin{array}{l}\text { The International Diabetes Federation (IDF) } \\
\text { documents the countries with the highest number } \\
\text { of diabetic patients: People's Republic of China, }\end{array}$ \\
& India, USA, Russia, Brazil \\
\hline
\end{tabular}

and Maternity and Infant Care; these terms were amended for the other databases.

"Contraception", "birth control", "intrauterine device/ IUD”, "Levonorgestrel intrauterine system/IUS”, "vaginal ring", "barrier method", "hormonal contraception", "contraceptive implant", "family planning", "reproductive health", "contraceptive counseling", "hormonal contraception", "sterilization", "contraceptive injection", "diabetes", "diabetes mellitus", "type 1 diabetes", "type 2 diabetes", "women with diabetes".

The terms were searched separately and in combination. References given in the listed papers were searched for papers that did not appear on any of the databases searched. The search was limited to studies in the English language. Articles that addressed the issues of contraception use in women with diabetes were qualified for inclusion in the review. The search yielded 1,837 results; most of these were duplicates due to the combination of search terms within the different databases. The titles and abstracts of 1,837 studies were screened, and those articles whose eligibility could not be determined from their titles and abstracts alone were read in full. The full text of 28 studies was read and 17 studies were found to be relevant. The relevant studies included reviews and primary quantitative and qualitative research studies.

\section{Facing the challenges}

DM can lead to numerous complications with long-term health implications on vascular, neurological, renal, and ophthalmic functions. ${ }^{5}$ From a reproductive perspective, poor glycemic control at the time of conception and during pregnancy increases the chance of adverse pregnancy outcomes including stillbirth, congenital abnormalities, and perinatal mortality. ${ }^{6}$ Neonates born to women with DM are "five times as likely to be stillborn, three times as likely to die in their first months of life, and twice as likely to have a major congenital anomaly". ${ }^{7}$ The UK Mothers and Babies: Reducing Risk through Audits and Confidential Enquiries across the UK (MBRRACE) report ${ }^{8}$ highlights other risks associated with diabetes and pregnancy, namely, hypoglycemia and diabetic ketoacidosis, and the importance of preconception advice and glycemic control with an $\mathrm{HbA}_{1 \mathrm{c}}<10 \%$.

In order to minimize complications, safe and effective contraception to prevent unintended pregnancy and preconception care is paramount for all women with DM. ${ }^{9}$ Monitoring and improving maternal health whilst encouraging adequate inter-pregnancy spacing and the use of effective contraception forms an integral part of pre-pregnancy care. ${ }^{1}$ This is a challenge for health care professionals as only half 
of the women with DM plan their pregnancies, ${ }^{7}$ and they can be reticent to ask for advice; ${ }^{10}$ a US National survey found that women with DM were less likely to be using contraception than women without DM. ${ }^{11}$

Lack of understanding about the high risks for unplanned pregnancy and diabetes and the need for effective contraception among women with DM are of concern. Cartwright et al ${ }^{12}$ published findings of an audit involving 100 women attending a diabetic clinic in UK. Findings revealed that two-thirds of women were not able to remember discussing pregnancy or contraception with a health care provider. The most common method of contraception (65\%) was the use of condoms and some women were unsure about suitable contraception. Those having had a pregnancy exhibited no additional knowledge regarding risks associated with pregnancy and DM.

Murphy et $\mathrm{al}^{13}$ explored the experiences of women with DM who did not attend pre-pregnancy care in the UK. Twentynine pregnant women with type 1 and type 2 DM participated in a qualitative study. Data were collected via semi-structured interviews and coded as part of thematic analysis. Women were found to have difficulty complying with contraceptive advice and the majority were not using reliable contraception prior to becoming pregnant. With regard to preconception health, although some women had experienced previous miscarriage, fetal anomaly, and stillbirth, they had however been reluctant to attend preconception care due to previous negative experiences, becoming pregnant quickly, having concerns relating to fertility, or simply due to practicalities associated with attending services.

The prevalence of DM is rising rapidly in the Middle Eastern countries and the developing world, ${ }^{2}$ and two studies from Iran have identified low use of contraception by women with DM. ${ }^{14,15}$ A mixed method cross-sectional study involving 200 women identified many pregnancy-related abnormalities and a lack of confidence in using contraceptives, with many women not using any form of contraception. Similar findings and low use of modern contraceptive methods with women mainly using coital withdrawal method were found in Iranian women with type 2 DM, hypertension, or obesity. ${ }^{15}$ Although the results of these studies may not be generalizable to different demographic communities, the importance of educating women with DM from all groups of society needs to be emphasized.

The challenge is to support women with type 1 and type 2 DM in making a choice of contraception based on their individual needs and associated risk factors. ${ }^{16}$ No restrictions for use of any contraceptive apply to women with GDM, but their propensity to develop type $2 \mathrm{DM}$ may need consideration.
Young people with unstable DM are of particular concern as they need reliable contraception in order to prevent high-risk unintended pregnancies.

\section{Providing contraceptive solutions}

Guidance about contraception needs to be offered holistically and a trusting relationship between practitioner and client is advocated. ${ }^{17}$ The importance of avoiding unintended pregnancy should be an integral part of diabetes education from adolescence onward discussed by diabetes health care providers. ${ }^{9}$ Choice of contraception should be made on the preference of the woman and individual risk factors identified such as the presence of vascular, nephropathy, neuropathy, or retinal disease. ${ }^{1,18}$ Choosing a safe and reliable method of contraception for a woman with DM needs careful consideration and practitioners need to make reference to the WHO Medical Eligibility Criteria for Contraceptive Use ${ }^{18}$ as outlined in Table 2.

Efficacy must be assured and the chosen method must not induce diabetic-related risks such as thromboembolic and cardiovascular complications. ${ }^{19}$ Those with poorly controlled diabetes and obese women may need to be targeted as they often fall victim to associated risks by not seeking advice and support. ${ }^{1}$

\section{Combined hormonal contraception}

Combined hormonal contraception (CHC) methods include the administration of estrogen and progestogen. Examples of $\mathrm{CHC}$ include combined contraceptive pills, transdermal contraceptive patches, combined vaginal rings, and combined injectable contraception. Historically, women with diabetes were denied $\mathrm{CHC}$ due to concerns about the effect on carbohydrate and lipid metabolism. ${ }^{20}$ Recent data highlight the safety of CHC not only in relation to women with DM without complications, but in not inducing diabetes in otherwise healthy individuals. ${ }^{1,20,21}$

A recent review of evidence ${ }^{1}$ emphasized that $\mathrm{CHC}$ containing less than $35 \mu \mathrm{g}$ of ethinyl-estradiol did not alter blood glucose concentrations and insulin secretion. In relation to

Table 2 WHO Medical Eligibility Criteria for Contraceptive Use (20I5)

\begin{tabular}{ll}
\hline Category & With clinical judgment \\
\hline & Use method in any circumstance \\
3 & Generally use method \\
& $\begin{array}{l}\text { Use of method not usually recommended unless other } \\
\text { more appropriate methods are not available or not } \\
\text { acceptable }\end{array}$ \\
4 & Method not to be used \\
\hline
\end{tabular}

Note: Reprinted from WHO. WHO Medical Eligibility Criteria for Contraceptive Use; Geneva: 2015. Available from: http://www.who.int/reproductivehealth/publications/ family_planning/mec-wheel-5th/en/. Accessed August 19, 2015. ${ }^{8}$ Copyright $\odot 2015$. 
diabetic retinopathy, macular edema, and nephropathy, there was no increase in risk or acceleration of disease in women taking oral CHC. ${ }^{1,19}$ The WHO Medical Eligibility Criteria for Contraceptive $\mathrm{Use}^{18}$ states (Table 3 ) that for any diabetic patient without cardiovascular or microvascular complications, advantages of using the method generally outweigh the theoretical or proven risks, and Category 2 applies for $\mathrm{CHC}$.

The results of a Cochrane review of randomized controlled trials by Visser et $\mathrm{al}^{21}$ was inconclusive in determining whether hormonal contraception affected carbohydrate and lipid metabolism and long-term complications in women with DM. The French Society of Endocrinology expert group, ${ }^{22}$ however, undertook a comprehensive review of evidence and pathophysiological data, as to the safe contraceptive options for diabetic women with risk factors, and concluded that from a hormonal perspective extreme caution is recommended as risk factors often do not occur in isolation. Gourdy ${ }^{1}$ in his review supports this and suggests that $\mathrm{CHC}$ with estrogens and progestogen should be avoided whatever their route of administration in women with DM who have microvascular complications such as severe retinopathy (ischemic or proliferative), active macular edema, or nephropathy with persistent proteinuria. In cases of DM complicated by cardiovascular or microvascular disease, the risks of prescribing $\mathrm{CHC}$ outweigh any advantages and a WHO Medical Eligibility Criteria (MEC) $3 / 4^{18}$ would be awarded. ${ }^{1,22,23} \mathrm{CHC}$ is also unlikely to be suitable for women with risk factors such as smoking, obesity, or hypertension due to increased risk of venous thromboembolism, myocardial infarction, and stroke. ${ }^{17,23}$

\section{Progestogen oral hormonal contraception}

The progestogen-only pill (POP) is regarded as a safe option for women with DM of any age with or without complications.

Table 3 Contraceptive methods and diabetes

\begin{tabular}{lllllll}
\hline Condition & CHC & POP & $\begin{array}{l}\text { DMPA/ } \\
\text { NET-EN }\end{array}$ & IMP & $\begin{array}{l}\text { Cu } \\
\text { IUD }\end{array}$ & IUS \\
\hline $\begin{array}{l}\text { History of gestational } \\
\text { diabetes }\end{array}$ & $\mathrm{I}$ & $\mathrm{I}$ & $\mathrm{I}$ & $\mathrm{I}$ & $\mathrm{I}$ & $\mathrm{I}$ \\
Nonvascular disease & & & & & & \\
$\begin{array}{l}\text { i. Noninsulin dependent } \\
\text { ii. Insulin dependent }\end{array}$ & 2 & 2 & 2 & 2 & $\mathrm{I}$ & 2 \\
Neuropathy/retinopathy/ & $3 / 4$ & 2 & 3 & 2 & $\mathrm{I}$ & 2 \\
neuropathy & & 2 & 2 & $\mathrm{I}$ & 2 \\
Other vascular disease & $3 / 4$ & 2 & 3 & 2 & $\mathrm{I}$ & 2 \\
\hline
\end{tabular}

Abbreviations: $\mathrm{CHC}$, combined hormonal contraception; $\mathrm{Cu}$ IUD, copper intrauterine device; DMPA/NET-EN, medroxyprogesterone acetate/Norethisterone enanthate; IMP, implants; POP, progestogen-only pill; IUS, intrauterine system. Note: Reprinted from WHO. WHO Medical Eligibility Criteria for Contraceptive Use; Geneva: 2015. Available from: http://www.who.int/reproductivehealth/publications/ family_planning/mec-wheel-5th/en/. Accessed August 19, 2015. ${ }^{18}$ Copyright (c) 2015.
Advantages of using the method generally outweigh the theoretical or proven risks (WHO MEC $2^{18}$ ). The POP needs to be taken within a daily set time period but compliance is likely to be good if scheduled with routine insulin or oral hypoglycemic medication. Desogestrel has the benefit of inhibiting ovulation in $97 \%$ of cases and a 12 hours dosing period, ${ }^{24}$ and therefore may be a more reliable choice especially for younger people with DM.

\section{Long-acting reversible contraception}

Long-acting reversible contraceptives (LARC) are not memory-dependent and have high efficacy, which can make them a good choice for women with DM. Methods include copper intrauterine device (IUD), intrauterine system (IUS), progestogen-only injectable contraceptives, progestogen-only subdermal implants, and combined vaginal ring. The combined vaginal ring has been included under $\mathrm{CHC}$ within this review. Despite their safety and effectiveness, LARCs are generally underutilized with only $15.5 \%$ of women worldwide using IUDs, and only 3.4\% using subdermal implants. ${ }^{25}$ In the UK in 2008-2009, only $12 \%$ of women aged 16-49 years used LARC compared to $25 \%$ of women who used COC and $25 \%$ whose male partners used condoms. ${ }^{26}$

Goldstuck and Steyn ${ }^{27}$ published a systematic review focusing on the efficacy of the IUD and IUS for women with DM. Four hundred and ninety nine articles were screened and seven studies met the inclusion criteria. Analysis resulted in the reassurance that the copper IUD and IUS were suitable for women with type 1 and type 2 DM, although the IUS needed further monitoring from a metabolic perspective in relation to type $2 \mathrm{DM}$. WHO $\mathrm{MEC}^{18}$ agrees that the IUD is entirely suitable for women with type 1 or type 2 DM with no restriction for use, and the advantages of using IUS generally outweigh the theoretical or proven risks.

A Cochrane review by Visser et $\mathrm{al}^{21}$ focused on evidences from randomized controlled trials relating to hormonal as opposed to nonhormonal contraception in diabetic patients. Results concluded that there was limited evidence relating to the effect of hormonal contraception on diabetes. Although the quality of the data was inconclusive, it identified the POP and the IUS as not being contraindicated for women with $\mathrm{DM}$, whereas the $\mathrm{CHC}$ was identified as having a negligible effect on glucose stability.

Progestogen-only injectable contraceptives, such as medroxyprogesterone acetate and norethindrone enanthate, are suitable for women with DM without complications. ${ }^{26}$ Concerns regarding possible negative effect on lipid 
metabolism due to hypoestrogenic effects and reduced HDL levels mean that for women with complications, risk outweighs benefit (MEC 3/4), and POPs would only be administered following specialist advice. ${ }^{18}$ Injectable methods may also cause a possible delay returning to fertility, ${ }^{26}$ which may be unacceptable to women with DM who may prefer to have families as soon as possible before complications of DM arise.

Progestogen-only subdermal implants containing etonogestrel are suitable for women with diabetes, as per WHO MEC guidelines. ${ }^{18}$ Implants release a constant dose of progestogen reducing the potential for metabolic variation and maintaining steady blood-lipid ratios. ${ }^{28}$

\section{Barrier and natural methods of contraception}

Barrier methods include a range of contraceptives including diaphragms, cervical caps, as well as male and female condoms. Some involve using spermicide such as nonoxynol-9; however, there appear to be no studies contraindicating the use of spermicide from a diabetic perspective. The decision to use a barrier method comes down to personal acceptability and efficacy; a male condom, for example, is $98 \%$ effective with proper use, a female condom $95 \%$, and a diaphragm with spermicide is $92 \%-96 \%$ effective. ${ }^{29}$ Using barrier methods alone may be too unreliable in preventing pregnancy for women with DM, especially if she is trying to lose weight or reduce her $\mathrm{HbA}_{1 \mathrm{c}}$ levels. ${ }^{26}$ Male and female condoms may be best used to prevent sexually transmitted infections, in conjunction with a more effective contraceptive such as hormonal methods or IUD.

Fertility awareness methods, although effective for some couples striving for a non-hormonal, more "natural" method of contraception, may not be a suitable choice for women with DM. In healthy women with a regular menstrual cycle and contemporaneous use, natural family planning is rated as being $99 \%$ effective. ${ }^{29}$ Women with DM have a potentially increased incidence of erratic menstrual cycles $^{30}$ that may affect efficacy and lead to an increased failure rate. ${ }^{31}$

\section{Male and female sterilization}

The failure rate for female sterilization is one in 200 and one in 2,000 for men following a vasectomy. ${ }^{29}$ Since both procedures involve an operative intervention, however, minor, glycemic control needs consideration since it can affect the chances of acquiring a postoperative infection. ${ }^{32}$ In addition, the chances of hypoglycaemia and ketoacidosis occurring, increase post surgery complications. ${ }^{33}$ Guidance from a diabetic physician regarding the safest operative location as well as prophylactic antibiotics are advisable if a couple decides that sterilization is the right choice. ${ }^{18}$

\section{Emergency contraception}

Emergency contraception provides all women with additional choice for prevention of pregnancy. The global rate of unplanned pregnancy in 2012 was 53 per 1,000 women aged between 15 years and 44 years. ${ }^{34}$ Emergency contraception may be required for multiple reasons including failure to use a contraceptive correctly and following sexual abuse or rape. Women with $\mathrm{DM}$ at risk and not wanting to be pregnant are advised to seek emergency contraception at the earliest opportunity. From a diabetic perspective, there are no contraindications to taking emergency progestogenonly contraceptive pills within the recommended time frame or to the use of the copper-bearing IUD within 5 days of unprotected intercourse or when ovulation is known, no more than 5 days after ovulation. ${ }^{35}$ According to the WHO MEC guidelines, ${ }^{18}$ the benefit of using emergency contraception outweighs the risk, even where there is severe vascular disease.

\section{Key solutions}

Our review has highlighted that women with DM need to use a reliable method of contraception in order to avoid high-risk unintended pregnancies. Women without complications can choose from the full range of methods that are available; ${ }^{18}$ however, studies have shown that they are less likely to use contraception or to seek advice. ${ }^{10,11}$ To improve pregnancy outcomes, women with DM need to be made aware of fertility and contraception advice services available within both acute and primary care.

Taking the earlier discussions into account, it is of concern that not all women with DM appear to understand the importance of contraceptive and/or preconception well-being from a diabetic perspective. Contraception messages in the diabetic female population need to be reinforced. ${ }^{12-15}$

There is a need for a more integrated approach to diabetes and reproductive health with improved communication between women with DM and their health care providers. ${ }^{13}$ Diabetes health professionals need to discuss contraception with women and referral to a contraceptive specialist for advice may be advisable. ${ }^{22,36}$

UK guidelines ${ }^{16}$ emphasize that women should choose their own contraceptive method, based on guidelines and taking account of any risks. In order to make appropriate choices, women with DM need to be provided with up-todate research-based evidence regarding suitable methods. 
The effectiveness of barrier methods and oral contraception is often compromised due to inconsistent use, ${ }^{26}$ and LARC methods, if suitable, will provide better efficacy. ${ }^{26}$

Women with DM need to plan any pregnancies and use reliable contraception until their diabetic health care provider confirms a satisfactory $\mathrm{HbA}_{1 \mathrm{c}}$ result, ideally below $6.5 \%{ }^{9}$ Women should be advised to maintain their "fasting blood glucose between 3.5 and $5.9 \mathrm{mmol} / \mathrm{L}$ and their 1 -hour postprandial blood glucose below $7.8 \mathrm{mmol} / \mathrm{L}$ if this is safely achievable". ${ }^{37}$ They do, however, need to be aware of the potential risk of hypoglycemic episodes and the appropriate treatment required. Women with an $\mathrm{HbA}_{1 \mathrm{c}}$ level above $86 \mathrm{mmol} / \mathrm{mol}(10 \%)$ are advised not to become pregnant due to associated risks. ${ }^{9}$ Before discontinuing contraception,

Table 4 Summary of recommendations

Individualized, specialist contraceptive advice

Contraceptive guidance needs to be offered holistically; a trusting

relationship between practitioner and client is advocated. ${ }^{17}$

Practitioners need to make reference to the WHO Medical Eligibility

Criteria for Contraceptive Use. ${ }^{18}$

Women should be informed of all methods available including LARC

methods which have higher efficacy. ${ }^{26}$

Contraceptive choices for women with diabetes

Recent data highlight the safety of hormonal contraception in relation

to women with DM without complications of cardiovascular or

microvascular risk factors. ${ }^{18}$

The copper IUD and IUS are suitable for women with type I and type 2 DM (MEC I/2). ${ }^{18}$

Injectable contraceptives (medroxyprogesterone acetate) are not contraindicated for healthy diabetic women (MEC 2). ${ }^{26}$ Women at risk are recommended that they seek specialist advice (MEC 3). ${ }^{18}$ Contraceptive implants are suitable for women with diabetes (MEC 2). ${ }^{18}$ Natural and barrier methods of contraception are not contraindicated for women looking for a nonhormonal method of birth control. Male and female sterilization remains an option; however, infection control is paramount ${ }^{32}$ and specialist referral is recommended. Emergency contraceptive pills and the copper IUD are suitable for all women with DM to prevent unintended pregnancy. ${ }^{18}$

Preconception care

Effective contraception is paramount for women exhibiting poor glycemic control. ${ }^{6}$

Women with diabetes have increased risks of adverse pregnancy outcomes: Fivefold increased risk of stillbirth, threefold increased risk of perinatal mortality, and twofold increased risk of fetal congenital anomaly. ${ }^{7}$ Contraceptive care prior to pregnancy can benefit optimal prepregnancy weight, stabilize glycemic control, ensure safety of medication, and provide baseline levels for monitoring retinal and renal functions. ${ }^{9}$ Contraception is advised until $\mathrm{HbA}_{\mathrm{Ic}}$ is below $6.5 \% .{ }^{9}$ Women whose $\mathrm{HbA}_{\mathrm{lc}}$ level is above $86 \mathrm{mmol} / \mathrm{mol}(\mathrm{I} 0 \%)$ are advised not to get pregnant. $^{9}$

Women with DM need to be made aware of fertility advice services available within both acute and primary care. ${ }^{9}$

Abbreviations: LARC, long-acting reversible contraceptives; DM, diabetes mellitus; IUD, intrauterine device; IUS, intrauterine system; MEC, Medical Eligibility Criteria; WHO, World Health Organization. a comprehensive drug assessment should be made to review and change medication that is potentially teratogenic, such as ACE inhibitors and certain insulins. ${ }^{9,38}$ In addition, women should be offered a renal assessment including measurement of microalbuminuria and retinal assessment to exclude progressive disease. ${ }^{9,38}$

Support and education prior to pregnancy, therefore, benefits by ensuring an optimal pre-pregnancy weight, stabilizing glycemic control, ensuring safety of medication, and providing baseline levels for monitoring retinal and renal functions. ${ }^{9,38}$ Delay of pregnancy to achieve favorable glycemic control has been found to prevent poor maternal and neonatal outcomes, ${ }^{6}$ and in most cases, maintaining an optimal inter-pregnancy interval reduces adverse birth outcomes and decreases maternal morbidity from a physical and psychological perspective..$^{39,40}$

Monitoring and improving health while encouraging adequate use of effective contraception forms an integral part of contraceptive care. Follow-up for women with DM using hormonal contraception is based on routine clinical practice of blood pressure and body mass index measurements, but baseline lipid profiles may need assessment, especially if injectable contraception is used, and blood glucose levels as relevant. ${ }^{19}$

In conclusion, the prevalence of DM in women of reproductive age is rising. Effective contraception is required in order to prevent unintended pregnancy as uncontrolled glucose levels at conception leads to poor maternal and perinatal outcomes. Contraceptive choices need to be made by women in conjunction with evidence-based research (Table 4). Women with DM without complications have multiple choices for contraception and those with complications may need specialist advice. Communication between health professionals and women with DM needs improving in relation to promoting reproductive health and contraception. A trusting, nonjudgmental approach and a positive relationship are important. This will enable the discussion of risks and benefits of contraceptive methods and provision of advice dedicated to improving overall health and well-being.

\section{Disclosure}

The authors report no conflicts of interest in this work.

\section{References}

1. Gourdy P. Diabetes and oral contraception. Best Pract Res Clin Endocrinol Metab. 2013;27:67-76.

2. International Diabetes Federation (IDF). IDF Diabetes Atlas. 6th ed; International Diabetes Federation: 2013. Available from: https://www. idf.org/sites/default/files/EN_6E_Atlas_Full_0.pdf. Accessed August 19, 2015. 
3. Dunlop AL, Jack BW, Bottalico JN. The clinical content of preconception care: women with chronic medical conditions. Am J Obstet Gynaecol. 2008;199:(6 Suppl B):S310-S327.

4. Diabetes UK. Diabetes: Facts and Stats. Version 4. London: Diabetes UK; Revised 2015.

5. Diabetes UK. Diabetes in the UK: Key Statistics on Diabetes. London: Diabetes UK; 2012. Available from: http://www.diabetes.org.uk/Docu ments/Reports/Diabetes-in-the-UK-2012.pdf. Accessed August 17, 2015.

6. Schwarz EB, Postlethwaite D, Yun-Yi Hung, Lantzman E, Armstrong MA, Horberg MA. Provision of contraceptive services to women with diabetes mellitus. J Gen Intern Med. 2011;27(2):196-201.

7. CEMACH. Diabetes in pregnancy: are we providing the best care? Findings of a national enquiry. London: Confidential Enquiry into Maternal and Child Health; 2007. Available from: http://bit.ly/ cemach2007. Accessed 31 July, 2015.

8. Knight M, Kenyon S, Brocklehurst P, Neilson J, Shakespeare J, Kurinczuk JJ, editors; On behalf of MBRRACE-UK. Saving Lives, Improving Mothers' Care - Lessons Learned to Inform Future Maternity Care from the UK and Ireland Confidential Enquiries into Maternal Deaths and Morbidity 2009-2012. Oxford: National Perinatal Epidemiology Unit, University of Oxford; 2014

9. National Institute for Health and Care Excellence (NICE). Management of Diabetes and Its Complications from Preconception to the Postnatal Period, (NG3). London: NICE; 2015.

10. Shawe J, Smith P, Stephenson J. Use of contraception by women with type 1 or type 2 diabetes mellitus: 'it's funny that nobody really spoke to me about it'. Eur J Contracept Reprod Health Care. 2011; 16(5):350-358.

11. Vahratian, A. Family-planning practices among women with diabetes and overweight and obese women in the 2002 National Survey for Family Growth. Diabetes Care. 2009;32:1026-1031.

12. Cartwright A, Wallymahmed M, Macfarlane I, Casson I. What do women with diabetes know about pregnancy and contraception? Pract Diabetes Int. 2009;26(6):238-242.

13. Murphy HR, Temple RC, Ballt VE, et al. Education and psychological aspects. Personal experiences of women with diabetes who do not attend pre-pregnancy care. Diabetic Med. 2010;27:92-100.

14. Aliasgharzadeh A, Aghamohammadzadeh N, Mobasseri M, et al. Study of contraception status of female diabetic patients in childbearing years. Res J Biol Sci. 2008;3(7):710-715.

15. Nojomi M, Morrovatdar N, Davoudi F, Hosseini S. Contraceptive use by Iranian women with hypertension, diabetes and obesity. East Mediterr Health J. 2013;19(7):638-643.

16. Faculty of Sexual and Reproductive Health Care. UK Medical Eligibility Criteria for Contraceptive Use (UKMEC 2009); London: 2009. Available from: http://www.fsrh.org/pdfs/UKMEC2009.pdf. Accessed February 13, 2015.

17. Shawe J. Contraceptive choices for women with diabetes'. Primary Care Women's Health Journal. 2013; 5(1):29-32.

18. WHO. WHO Medical Eligibility Criteria for Contraceptive Use; Geneva: 2015. Available from: http://www.who.int/reproductivehealth/ publications/family_planning/mec-wheel-5th/en/. Accessed August 19, 2015.

19. Skouby SO. Hormonal contraception in obesity, the metabolic syndrome and diabetes. Ann N Y Acad Sci. 2010;1205:240-244.

20. Pallardo LF, Cano A, Cristobal I, Blanco MA, Lozano M, Lete I. Hormonal contraception and diabetes. Clin Med Insights Women's Health. 2012;5:53-63.

21. Visser J, Snel VJ, Van Vilet HA. Hormonal versus non-hormonal contraceptives in women with diabetes mellitus type 1 and 2 (review). Cochrane Database Syst Rev. 2013;3:CD003990.
22. Gourdy P, Bachelot A, Catteau-Jonard S, et al. Hormonal contraception in women at risk of vascular and metabolic disorders: Guidelines of the French Society of Endocrinology. Ann Endocrinol. 2012;73:469-487.

23. Faculty of Sexual and Reproductive Health Care (FSRH). Member's Enquiry Response EdME0040. London: FSRH; 2014.

24. Faculty of Sexual and Reproductive Health Care (FSRH). Progestogen Only Pills. London:FSRH; 2008, updated 2009.

25. Blumenthal PD, Voedisch A, Gemzell-Danielsson K. Strategies to prevent unintended pregnancy: increasing use of long-acting reversible contraception. Hum Reprod Update. 2010;17(1):121-137.

26. National Institute for Health and Care Excellence (NICE). Guidance on Long-Acting Reversible Contraception (Update). London: NICE; 2014. Accessed from: http://www.nice.org.uk/CG30. Accessed August $19,2015$.

27. Goldstuck ND, Steyn PS. The intrauterine device in women with diabetes mellitus type I and II: a systematic review. ISRN Obstet Gynecol. 2013;2013:814062.

28. Diab KM, Zaki MM. Contraception in diabetic women: comparative metabolic study of Norplant, depot medroxyprogesterone acetate, low dose oral contraceptive pill and CuT380A. J Obstet Gynaecol Res.2000;26:17-26.

29. Family Planning Association (FPA). Advice on Choice of Contraceptive Method from The Family Planning Association; London: 2010. Available from: http://www.fpa.org.uk. Accessed August 19, 2015.

30. Snell-Bergeon JK, Dabelea D, Ogden LG, et al. Reproductive history and hormonal birth control use are associated with coronary calcium progression in women with type 1 diabetes mellitus. J Clin Endocrinol Metab. 2008;93(6):2142-2148.

31. Shawe JA. Pre-pregnancy care: a review of preconception care policy, guidelines and recommendations across six European countries. European Journal of Contraception and Reproductive Sexual Health. 2014;19(1):57-65.

32. Frisch A, Chandra P, Smiley D, Peng L, Rizzo M, Gatcliffe C. Prevalence and clinical outcome of hyperglycemia in the perioperative period in non-cardiac surgery. Diabetes Care, 2010;33:1783-1788.

33. National Health Service (NHS). Management of Adults with Diabetes Undergoing Surgery and Elective Procedures: Improving Standards. London: NHS; 2011. Accessed from: http://www.diabetologists-abcd. org.uk. Accessed August 4, 2015.

34. Sedgh G, Singh S, Hussain R. Intended and unintended pregnancies worldwide in 2012 and recent trends. Stud Fam Plann. 2014;45(3):301-314.

35. Faculty of Sexual and Reproductive Health Care (FSRH). Combined Hormonal Contraception. London: FSRH; 2011, updated 2012.

36. Shawe J, Mulnier H, Nicholls P, Lawrenson R. Use of hormonal contraceptive methods by women with diabetes. Prim Care Diabetes. 2008;2:195-199.

37. Mugglestone MA. Management of diabetes from preconception to the postnatal period: summary of NICE guidance. $B M J$ 2008;336:714-717.

38. National Institute for Health and Clinical Excellence (NICE) Clinical Guideline 63. Diabetes in Pregnancy: Management of Diabetes and Its Complication from Preconception to the Postnatal Period. London: NICE; 2008 Available from: http://www.nice.org.uk/nicemedia/pdf/ CG063Guidance.pdf. Accessed August 17, 2015.

39. Ball SJ, Pereira G, Jacoby P, de Klerk N, Stanley FJ. Re-evaluation of link between interpregnancy interval and adverse birth outcomes: retrospective cohort study matching two intervals per mother. BMJ. 2014;349:g4333.

40. Cleland J, Conde-Agudelo H, Peterson J, Ross AT. Contraception and health. Lancet. 2012;380(9837):149. 


\section{Publish your work in this journal}

Open Access Journal of Contraception is an international, peerreviewed, open access, online journal, publishing original research, reports, reviews and commentaries on all areas of contraception. In addition to clinical research, demographics and health-related aspects, the journal welcomes new findings in animal and preclinical studies

relating to understanding the biological mechanisms and practical development of new contraceptive agents. The manuscript management system is completely online and includes a very quick and fair peer-review system. Visit http://www.dovepress.com/testimonials.php to read real quotes from published authors.

Submit your manuscript here: http://www.dovepress.com/open-access-journal-of-contraception-journal 